\title{
Le territoire entre politique de développement et attractivité
}

The Territory between Development Policy and Attractiveness

\section{Thomas Lamarche}

\section{(2) OpenEdition}

\section{Journals}

Édition électronique

URL : http://journals.openedition.org/edc/122

DOI : $10.4000 /$ edc. 122

ISSN : 2101-0366

Éditeur

Université de Lille

Édition imprimée

Date de publication : 1 décembre 2003

ISSN : 1270-6841

Référence électronique

Thomas Lamarche, «Le territoire entre politique de développement et attractivité », Études de

communication [En ligne], 26 | 2003, mis en ligne le 13 octobre 2008, consulté le 10 décembre 2020

URL : http://journals.openedition.org/edc/122 ; DOI : https://doi.org/10.4000/edc.122

Ce document a été généré automatiquement le 10 décembre 2020.

(c) Tous droits réservés 


\title{
Le territoire entre politique de développement et attractivité
}

The Territory between Development Policy and Attractiveness

\author{
Thomas Lamarche
}

1 La longue période de restructuration industrielle qui débute dans les années 1970 remet en cause les institutions et mécanismes fondamentaux du fordisme. Une logique déréglementaire de mise en concurrence et de mise en management atteint des secteurs entiers : banque-finance, énergie, services publics (télécom, transport, services urbains, bientôt éducation, santé...). La crise de l'État débouche sur une crise du territoire, notamment de l'État-nation qui s'interroge sur sa capacité à figurer comme échelon de pouvoir pertinent dans une économie mondialisée. L'État-providence keynésien issu de construits sociaux et politiques nationaux était en lui-même territorialisé (Boyer, 1986). Deux composantes du fordisme sont touchées dans le processus de mondialisation : l'État comme mode principal d'intervention publique et le territoire national comme espace d'élaboration des régularités économiques.

2 Le territoire dont il s'agit ici de traiter est, dans un premier temps, le territoire national. La littérature concernant le territoire considère le plus souvent cette notion dans son acception locale. Pourtant, si «le territoire devient un problème dès lors que l'on constate que soumis à des forces historiques identiques [...] des territoires différents produisent des dynamiques différentes $»^{1}$, alors l'intérêt porté au territoire national est une continuité logique.

3 Internationalisation de l'économie et crise de l'État modifient les rapports de force de telle façon que certains auteurs dressent le contour d'une forme de dépérissement de l'État-nation (Michalet, 1999). La dynamique internationale est davantage menée par les firmes que par les États-nations ${ }^{2}$, et la régulation des activités économiques échappe au niveau national car les regroupements territoriaux (Union européenne, ALENA...) et les institutions internationales (OMC, FMI...) tentent de former des espaces de régulation. 


\section{Éléments d'une déréglementation du territoire}

4 La logique déréglementaire qui transforme l'organisation de plusieurs secteurs économiques atteint le territoire en tant qu'action publique. La mutation des politiques d'aménagement, puis de développement des territoires est liée à une manière de penser le territoire comme une offre comme les autres. Comme le disent certains professionnels $\mathrm{du}$ marketing territorial, "le territoire est un produit comme les autres $»^{3}$. Jacques Séguéla avait, dans une logique similaire, présenté le vote comme un acte de consommation comme les autres. Ce qui relève d'une même forme de simplification.

5 À la traversée du désert de l'aménagement du territoire, laissé pour compte pendant les années de crise industrielle, se substitue la montée de la logique d'attractivité.

Crise de l'aménagement du territoire et montée de la logique d'attractivité

7 La notion d'attractivité indique la capacité d'un territoire à attirer des investissements étrangers. La place prise par cette notion s'explique par le glissement de l'économie internationale vers l'économie industrielle. Dans la filiation ricardienne, l'économie internationale s'est longtemps basée sur la notion d'avantage comparatif. Même rénovée, cette logique libérale de spécialisation restait fortement basée sur les ressources (dotations initiales) et ainsi les avantages comparatifs étaient figés.

8 Avec la construction de la notion d'avantages concurrentiels, Michael Porter (1990) transfert aux territoires une logique industrielle de construction d'atouts dans une relation de concurrence sur un marché. Les avantages ne sont plus donnés, ce sont des construits politiques (politique publique et politique d'entreprise).

9 Les firmes transnationales acquièrent une place d'acteur prépondérant car elles décident des espaces où investir. Leur avantage concurrentiel résultera des avantages concurrentiels développés par les territoires.

10 Cette situation se tend lorsque la dimension financière de la mondialisation s'affirme. Dans les années 1990, ce n'est plus essentiellement la recherche d'un profit d'activité qui anime la firme mais un profit financier (issu de jeux sur l'échange de capitaux par opposition à la production de marchandises). Pris dans ce raisonnement, les territoires se livrent à une concurrence pour attirer un capital international qui est devenu incontestablement plus mobile. La mondialisation est un choix politique, "les gouvernements ont jeté par-dessus bord la base de légitimité de leur intervention dans l'économie en choisissant le néo-libéralisme contre le keynésianisme [...], ils se sont mis à la remorque des grandes multinationales » (Michalet, 1999, p. 129).

11 Dans ce cadre, les IDE (Investissements directs à l'étranger) constituent un indicateur majeur de la capacité des territoires à attirer les capitaux. Ces investissements sont la traduction d'un enchaînement particulier qui voit progressivement les territoires se résoudre, voire se convertir, à pratiquer une politique systématique visant à attirer les capitaux.

12 La marge de manœuvre des pouvoirs publics est profondément transformée. L'existence d'une multiplicité de niveaux de compétence politique (de la commune à l'institution supranationale), la modification des modalités de l'action publique (retrait des politiques budgétaires au profit d'une action monétaire) et enfin la montée d'une pensée économiciste favorisent une analyse des territoires en termes de rivalité. La marge de manœuvre des pouvoirs territoriaux est alors considérée en terme de renforcement de 
ces avantages concurrentiels. La séduction des nations (titre de l'ouvrage de Michalet) devient un principe d'action publique faisant primer l'attraction des capitaux (et secondairement du travail) dans un schéma de rivalité entre territoires. On distingue trois modèles qui peuvent s'interpénétrer :

- Valorisation quasi-publicitaire du territoire dans une perspective de marketing territorial. Le territoire est construit sur la base d'une image, l'enjeu auprès de différentes cibles s'insère dans la logique de la marque. Au printemps 2003, la campagne Basse Normandie passe sur de nombreux supports (radio, magazines, affichage).

L'association de la région à un haut niveau de qualité des produits (biens de luxe) est l'enjeu du volet publicitaire de ce marketing territorial. La dimension image est ici prise en charge par une agence de communication de la même façon que la construction d'une marque.

- Mise en avant de la compétitivité prix du territoire. Le lien compétitivitéattractivité conduit à prouver aux investisseurs potentiels un bon rapport qualitéprix. Ces deux notions, qualité d'une offre territoriale et prix de ce territoire, sont sujettes à interprétations multiples. Il ressort de ce modèle une focalisation sur les coûts directs pour l'investisseur (coûts sociaux liés au travail et fiscalité). On reviendra sur la composante prix qui est le moyen d'une politique d'attractivité apparente (construction d'une image-prix): zone franche, défiscalisation temporaire...

- Une politique de valorisation des compétences du territoire. Le développement de biens publics met en avant les infrastructures en communication (routes, télécom), la formation et la facilité d'accès aux technologies qui deviennent la condition de l'attractivité du territoire. Il y a là un travail sur le développement endogène que l'on verra en dernière partie. L'essentiel n'est plus le prix mais la ressource disponible au sein du territoire.

Ces trois composantes constituent des options qui sont couramment associées. Les deux premières forment un ensemble assez identifiable, cependant on retrouve des traces de la troisième composante, plus qualitative, qui peut s'associer à une dimension publicitaire notamment.

La stratégie d'image se nourrit d'apports des pratiques managériales. Il s'agit de faire comme si les bonnes pratiques de gestion pouvaient passer de l'entreprise au territoire. Le rapport parlementaire de Michel Charzat ${ }^{4}$ qui calque sa rédaction sur un plan marketing (force/faiblesse/opportunité/menace) et agence des propositions à la manière d'un plan de communication est emblématique de l'infiltration d'une pensée managériale du territoire.

Un des effets de la managérialisation est la transformation des agences (nationales et régionales) de développement des territoires en agences de promotion ${ }^{5}$. En entrant dans le jeu de la mise en concurrence, les territoires cherchent à satisfaire les attentes et besoins des investisseurs internationaux, il s'agit de faire mieux que les autres territoires rivaux... Ce faisant, les institutions locales construisent des « avantages spécifiques et des externalités positives ciblées » (Héraud et Kahn, 2002). Ces externalités sont par exemple des infrastructures publiques de transport, de communication, de distribution d'énergie... qui participent finalement à la production de profits privés. Dans le même ordre d'idée, les pratiques des agences de promotion, lorsqu'elles instruisent les dossiers d'insertion locale des investisseurs étrangers, sont une importante activité de service et d'ingénierie... que le public prend en charge. 


\section{La pensée d'un territoire en concurrence : omniprésence d'une logique « par les coûts »}

16 Un effet pervers associé aux politiques monétaristes de désinflation compétitive des années 1980 et 1990 est que la lutte contre l'inflation reste prioritaire mais non coopérative. Il s'agit pour chaque pays de faire mieux que ses partenaires européens : faire moins d'inflation, c'est améliorer sa compétitivité prix. La politique économique de l'Union européenne apparaît plus faite de compétition que d'harmonisation.

17 Lorsque le territoire se pense en concurrence, cela peut le conduire à une surenchère caractéristique d'une situation concurrentielle. Le cas des facteurs mesurables financièrement par l'investisseur mérite une attention particulière car ils se différencient des caractéristiques qualitatives plus diffuses (niveau de formation du bassin de population, performance des infrastructures...).

18 La course à l'avantage financier que mènent certains investisseurs incite les territoires à produire différentes formes de dumping. Le dumping auquel se livrent les autorités publiques territoriales marque un comportement de rivalité/surenchère et une focalisation sur les critères de coût. On peut synthétiquement définir deux catégories d'actions issues d'une logique de coûts: l'attractivité apparente et les aides (qui reviennent à diminuer le coût d'accès pour la firme arrivante).

19 Les politiques d'attractivité apparente constituent des formes de dumping en favorisant un point de vue comptable, ce qui conduit à focaliser sur les charges que supportent les firmes : charges sociales et charges fiscales. Deux récents rapports parlementaires sont éloquents sur le traitement de la question par la représentation nationale : le rapport de Michel Charzat (PS) en 2001, déjà évoqué, et celui d'olivier Dassault (UMP) en 20036.

20 Le dumping social met en avant la flexibilité du travail, le bas niveau des salaires et adresse ainsi aux entreprises une promesse qui se mesure en terme de coût (le faible coût du travail peu qualifié) et en terme de réversibilité (liée aux formes flexibles du contrat de travail). Le modèle de la compétitivité prix est double : le territoire offre des ressources peu coûteuses, il est compétitif; et l'entreprise cherche à défendre une compétitivité par les coûts. Les exemples britannique et sud-est asiatique sont explicites : là prime un modèle de spécialisation (par le coût de la main d'œuvre) dont la justification théorique remonte à Ricardo.

21 Le dumping fiscal constitue une dimension moins présente mais régulièrement associée au dumping social. Le rapport d'olivier Dassault plaide ${ }^{7}$, au nom du déficit d'attractivité de la France, pour une réforme de l'ISF (Impôt de solidarité sur la fortune). On retrouve ici une antienne sur la fiscalité du capital : l'impôt sur le capital le fait fuir, il convient alors d'être attractif fiscalement. Actions fiscales et sociales convergent dans des propositions de déréglementation sociale (baisse des charges, nouveaux contrats de travail...).

L'implantation des sièges sociaux des grandes entreprises en Europe montre ainsi la capacité de certains territoires (ici des villes) à attirer les centres de direction, non pas seulement sur des critères de localisation de l'activité ou de proximité entre pairs... mais en fonction de facteurs fiscaux-financiers (non-exclusifs, évidemment). Alors que Londres et Bruxelles offrent une place d'entrée dans les réseaux principaux du pouvoir et de l'information, La Haye attire une part importante de sièges sociaux d'entreprises 
européennes non liées aux Pays-Bas par sa politique fiscale. Ainsi, parmi les différentes entités constitutives de la firme, les centres qui dégagent la plus value (par opposition courante avec les centres qui gèrent les différentes étapes de la production) sont eux aussi localisés-délocalisés sur critères fiscaux (les situations d'Alcatel, et dans une moindre mesure de STMicroelectonics, sont exemplaires de ce phénomène) ${ }^{8}$.

Enfin, si les territoires se livrent à une course à la subvention, aucune étude ne prouve l'effet direct de telles aides (Veltz, 2002). Les aides semblent d'une utilité finale, lorsqu'il y a hésitation entre deux localisations. Le choix de l'implantation d'un synchrotron en France a donné lieu à une vaste concurrence entre l'Ile-de-France et le Nord/Pas-deCalais, la victoire parisienne peuvant être attribuée à la subvention accordée en fin de négociation par le Conseil Régional'. Dans cette situation, la rivalité est infra-nationale et la logique d'aide intervient dans la décision finale.

\section{Un territoire exogène, pensé comme une ressource}

La logique ricardienne réinterprétée par le marketing territorial réduit les autorités locales à penser le territoire comme une ressource pour les grandes firmes mobiles. Cette analyse relève d'un présupposé issu d'une pensée libérale standard: les facteurs de production sont mobiles, ils font l'objet d'un mécanisme d'allocation (i.e. ils sont plus ou moins demandés) et la production de richesse est principalement organisée par la firme. Le territoire, immobile par définition, se réduit à une fonction de ressource mise à disposition de la firme transnationale qui gère une activité de production en fonction d'une nouvelle division internationale ${ }^{10}$.

Pourtant ces stratégies de globalisation qui animent un petit nombre de très grandes firmes ne sont qu'un des facteurs structurant le territoire. Elles agissent comme un facteur marquant (localisation et délocalisation du capital) et produisent une forme de résignation politique (symbolisée par une expression française ad hoc «contrainte extérieure ", qui résume une des formes d'exogénéisation du territoire).

La notion de stratégie de globalisation recouvre des stratégies industrielles particulières que ne peuvent mettre en place qu'un petit nombre de groupes industriels, elle suppose un certain seuil de développement (difficile à délimiter cependant) permettant de penser l'échelle mondiale. Dans le foisonnement de publications qui visent à caractériser la mondialisation, un clivage oppose le territoire vu comme une ressource (à adapter) et le territoire comme lui-même producteur de richesse. Les stratégies de globalisation correspondent à la première : ce sont des visions managériales de la mondialisation.

Les particularités locales des relations capital-travail permettent de produire des formes de rentes (ou d'avantages concurrentiels). L'opposition entre développement exogène basé sur l'attractivité de forces extérieures - et développement endogène - basé sur le soutien aux forces internes - a pour enjeu le partage de la rente entre les acteurs (notamment firmes transnationales d'une part et acteurs locaux d'autre part). Est-ce l'oligopole qui s'approprie la rente (et part quand la rentabilité baisse ou quand les aides s'arrêtent selon les effets d'aubaine) ou la rente est-elle (en partie au moins) appropriée par le territoire? 


\section{Le territoire face au capital ?} indirect. composent le territoire :

Une des principales caractéristiques du capitalisme patrimonial, selon François Chesnais (1997), est le rôle central des droits de propriété, et donc de ses détenteurs, aux dépens des autres parties prenantes. Deux phénomènes s'associent :

Accroissement du pouvoir de la grande entreprise, aux dépens des autres acteurs sociaux, ce qui ne peut se confondre avec le pouvoir du marché ;

Accroissement du pouvoir des propriétaires au sein de la firme.

Dans la technostructure décrite par Galbraith pour caractériser la firme des années de croissance, les stackholders (à l'époque essentiellement managers et salariés) étaient des acteurs centraux et le pouvoir des propriétaires du capital (les stockholders) était relativement réduit au profit de la structure elle-même.

Il convient de distinguer différentes politiques de détention d'actions qui reflètent deux tendances du capitalisme contemporain, shareholders et stockholders :

Acquisition d'actions liée à une stratégie industrielle. La propriété vise une part active (share) dans la production ;

Acquisition d'actions dans un but financier : constitution de plus-value financière

liée à la variation du cours boursier du titre (stock).

Ce détour par l'analyse de la firme permet de caractériser les logiques de mobilité qui animent les firmes transnationales. La mobilité du capital relève d'une question spatiale (IDE, localisation de la production...) et de logique de croissance externe (fusionacquisition, filialisation, participation...). La mobilité spatiale s'insère dans une logique productive alors que ce n'est pas toujours le cas pour la mobilité financière.

Le caractère exogène du développement territorial constitue une dimension de la conflictualité inhérente au capitalisme. Dans le statut de la Société Anonyme, les propriétaires du capital exercent un pouvoir direct, mais aussi, de façon croissante,

À la place du capitalisme historique où se confrontent capital et travail, apparaît une tension intégrant le territoire. Cela suppose d'identifier les acteurs non-mobiles qui

Le travail, salariat local, travailleurs indépendants, petits entrepreneurs, qui à des

degrés divers sont plus ou moins immobiles;

Pouvoirs publics, service publics et infrastructures elles-mêmes ;

Habitants, notamment fractions les moins mobiles des habitants (retraités...).

4 La thématique de la mobilité, très présente dans la construction européenne, vise à construire la fluidité des facteurs. L'enjeu de la mobilité (voire de la mobilisation, dans le sens de rendre mobile) des facteurs au sein de l'Union européenne constitue un puissant indicateur de la volonté de façonner un fonctionnement marchand pour toute sorte de ressources (faire circuler les salariés, les étudiants...).

\section{Localisation des investissements et évaluation de l'attractivité}

La mondialisation entre 1980 et 2000 n'est pas tant marquée par le développement commercial que par une logique financière, du fait des changements de propriété; 
l'internationalisation des services repose sur des transferts de capitaux et donc des prises de pouvoir local (Boltanski et Chiapello, 1999). En effet, l'exportation des services suppose différentes formes de localisations :

Implantation près des consommateurs finaux (service intégrant sa distribution); Développement d'infrastructures (implantation localisée des services publics en réseau) ;

Concentration dans des zones réduites, mais exerçant à l'échelle mondiale (conseil, finance, localisés à New York, la City, Tokyo).

L'extension internationale des firmes de services a conduit à de très importants transferts de propriété selon deux mouvements - privatisation et fusions-acquisitions ${ }^{11}$ - le premier entretenant le second.

L'attractivité induit un effet d'exogénéisation dans le sens où le lieu de décision (pouvoir du capital) et les lieux où ces décisions prennent effet sont déconnectés. Le territoire exogène est celui dont la richesse, le travail, l'activité économique résultent d'acteurs extérieurs. Relativisons ce critère exogène en rappelant que le capital, fut-il national, peut prendre toute sorte de décision contraire à l'intérêt de la population locale (la vague de licenciements qui marque ce début de siècle n'épargne pas les lieux d'origine du (apital) ${ }^{12}$.

Dès lors il n'y a pas seulement une question sur le développement exogène du territoire, mais plus fondamentalement sur le caractère hétéronome du capitalisme. L'hétéronomie, selon André Gorz (1988), qualifie la non maîtrise et la perte d'autonomie du sujet. L'individu (ou ici le territoire) est alors dirigé de l'extérieur, par une organisation préétablie. L'organisation bureaucratique produit une "hétéronomie programmée " (c'est l'organisation qui dirige l'action des individus), alors que le marché fonde une hétéronomie "non programmée». Ces deux phénomènes sont inhérents à l'approfondissement de la division du travail, notamment entre une composante immatérielle de conception-contrôle-protection juridique et une composante matérielle participant de moins en moins à la production de valeur.

L'hétéronomie constitue un puissant ressort critique du capitalisme, dépassant le cadre de l'analyse du territoire, mais permettant, ici, de situer un des enjeux de perte de pouvoir du local. Le système démocratique est finalement mis en péril par sa soumission à une décision externe elle-même issue d'un pouvoir privé et non d'une forme d'intégration internationale.

40 La reconnaissance du caractère plus ou moins attractif des territoires devient un enjeu fort puisqu'il s'agit pour les États de se mesurer les uns les autres, de se jauger... cela permet secondairement aux firmes de repérer les zones attractives. À l'instar des indicateurs boursiers et de la notation financière indiquant les performances des firmes, se développent des classements d'attractivité, qui formalisent une relation de concurrence entre les territoires. Deux polémiques récentes à propos d'indicateurs économiques éclairent leur rôle.

41 La première prend place début 2002 lors de la campagne pour les élections présidentielles en France. Eurostat publie un classement des performances économiques des 15 membres de l'Union européenne dans lequel la France est classée 12e (contre 3e en 1990). Cela nourrit une polémique autour du déclin de la position française, d'autant que les erreurs de calcul sont nombreuses ${ }^{13}$. Début 2003, Eurostat modère son jugement et corrige ses erreurs, la France se trouve au-dessus de la moyenne européenne à niveau égal de l'Italie, l'Allemagne et le Royaume-Uni. 

France à la trentième place ${ }^{14}$... large écho. convergence et d'attractivité.

La seconde s'est développée en 2002, elle fait ressortir le caractère normalisateur de tels classements. Le World Economic Forum, connu pour le Forum annuel organisé à Davos, produit un classement de la compétitivité des États. Or le classement 2002 relègue la

Deux situations distinctes, mais qui ont en commun d'avoir mis l'attractivité et la compétitivité de la France sur la sellette et d'avoir été reprises dans un argumentaire politique courant. Les débats sur la loi de finance 2003 à l'Assemblée Nationale y font un

L'enjeu de ces chiffres est lié à leur statut d'indicateur mais aussi au sens politique qui peut leur être attribué car, parallèlement au débat sur la perte d'attractivité, la France se situe parmi les 3 premiers pays du monde pour les IDE entrants. Ce qui mérite d'être pris en compte comme une indication d'attractivité effective (Veltz, 2002).

L'analyse d'un grand nombre de comparaisons internationales, menée par Boyer (2002), fait ressortir un autre travers de certaines études ou indicateurs. L'OCDE produit ainsi des analyses qui prennent la forme de benchmarking, c'est-à-dire de comparaison selon une méthode gestionnaire utilisée par les firmes, rendant le propos normatif et participant à l'assimilation des économies nationales aux firmes. De plus, la référence aux États-Unis conduit à faire du capitalisme américain la norme, en niant les variétés. L'intégration des États dans un univers concurrentiel mondial est l'enjeu de ces modèles normatifs de

\section{Le développement endogène du territoire}

En fin de compte, ce sont les acteurs locaux qui subissent et donc prennent en charge les interdépendances et les effets externes ${ }^{15}$, c'est localement que la faible durabilité du développement se mesure. La mobilité du capital (surtout financier) se rémunère sur l'immobilité d'autres facteurs (la terre, le travail...). La mondialisation renforce l'exogénéité (les décisions extérieures sont structurantes). Face à cela, nombre d'initiatives nationales/locales tentent d'endogénéiser le développement en soutenant les facteurs locaux, non-mobiles (innovations, formation, initiatives locales...).

Un important axe de renouvellement dans la pensée du post-fordisme provient d'une réflexion sur ses formes territoriales. En se basant sur la notion de district industriel décrit par Alfred Marshall à la fin du XIXe siècle, Piore et Sabel (1984) lient les nouvelles formes de production flexible à leur insertion dans le territoire. Leurs travaux coïncident avec les fructueuses descriptions du mode d'industrialisation de la troisième Italie $^{16}$ et engendrent une génération de réflexions sur le développement endogène ${ }^{17}$.

Des formes de concurrence-coopération, de relations basées sur la confiance, de liens affinitaires avec le local... sont soutenues à un niveau de pouvoir qui permet aux acteurs de se concerter, de se connaître. Par opposition au modèle de la firme mondiale qui produit de la décision et de l'organisation à distance, la construction locale de l'activité économique constitue un horizon où l'action du pouvoir local perdure. La dimension alternative de l'action économique locale provient de ce qu'elle permet la rencontre d'acteurs économiques (PME locales), d'acteurs publics (collectivités territoriales), en associant des formes d'autonomie du local.

La dimension politique sous-jacente de ces constructions se traduit par des propositions en terme de politique publique prenant conjointement en compte logique de 
développement territorial et gestion des externalités positives et négatives. Cette position est particulièrement manifeste chez Alain Lipietz ${ }^{18}$ et Bernard Pecqueur ${ }^{19}$ qui allient activité de recherche et pratique politique sur les questions de territoire. Cette dynamique conduit à intégrer la notion de développement durable aux recherches sur le territoire (Héraud et Kahn, 2002; Laganier, Villalba et Zuindeau, 2002). Districts et systèmes productifs locaux sont cependant parfois mythifiés car ils font apparaître une marge de manœuvre là où la mondialisation semble imposer une soumission à un ordre extérieur ${ }^{20}$.

Les stratégies de développement exogène répondent à la logique de spécialisation que développent nombre de firmes agissant à l'échelle mondiale. La firme fait ce qu'elle sait le mieux faire et externalise les domaines qui lui semblent secondaires. La stratégie exogène des territoires vise à attirer le capital en mettant en avant une particularité, qui se traduit comme avantage pour la firme. La stratégie de localisation des firmes est une géographie des coûts, mais de plus en plus également une géographie de l'organisation (Veltz, 1996). L'éclatement de la production réduit le pouvoir local des territoires : les filiales comme les sous-traitants sont soumis à une organisation exogène.

51 Le développement endogène consiste non plus à se baser sur l'attractivité mais à construire une base locale d'activité, en misant sur des facteurs non-mobiles (à l'inverse $\mathrm{du}$ capital financier). L'action publique (du local au supranational) vise à valoriser l'innovation, les initiatives locales, les facteurs endogènes spécifiques... Stratégies endogène et exogène ne se construisent pas de stricte façon antagoniste et n'existent pas à l'état pur, chaque territoire les associe de différentes manières. On peut, avec Zuindeau (2000), noter que le développement durable suppose un principe de non-rivalité qui est étranger à l'attractivité. En effet, une politique d'attractivité consiste à reporter une partie des charges sur des tiers (cf. le dumping, supra). Dans le domaine de l'environnement, des spécialisations se développent sur des productions polluantes ou à risque dans des pays à plus faible réglementation ; ces pays ne font pas payer les externalités... et attirent l'investissement par une rivalité non-durable.

Pourtant le fonctionnement des districts industriels garde une part d'ombre, il n'est pas réductible à une recette qui permettrait de créer du développement local. Bien qu'optimiste sur le sujet, Bernard Pecqueur considère qu'il n'existe pas de paradigme, pas de modèle " clé en main » (Pecqueur, 2000, p. 51). Les systèmes productifs locaux sont des construits longs qui ne résultent pas de façon déterministe d'une action politique uniforme. Ils reposent sur des facteurs que les économistes peinent à définir : culture commune, confiance et solidarité. Ainsi dans les districts, des échanges non monétaires ont une place décisive : partage d'informations technico-économiques (l'information devient ressource collective et ne sert pas à la compétitivité intra-district) ; partage d'expérience ; participation à la construction du savoir et des compétences en lien avec le système éducatif... autant d'ingrédients qu'une politique publique peut susciter.

Le développement endogène mise sur la pérennité des actions et non sur la recherche d'un avantage non durable lié à la mobilité du capital, il repose sur une forte part de collectif et de coopératif, sur un développement en réseau dans lequel s'illustrent des tissus de PME, alors que l'internationalisation des groupes les place en situation d'extériorité vis à vis du territoire. 


\section{BIBLIOGRAPHIE}

Benko, G. et Lipietz, A., (2000), La richesse des régions. La nouvelle géographie socio-économique, P.U.F.

Benko, G. et Lipietz, A., (1992), Les régions qui gagnent. Districts et réseaux : les nouveaux paradigmes de la géographie économique, P.U.F.

Boltanski, L. et Chiapello, E., (1999), Le nouvel esprit du capitalisme, Gallimard, nrf essais.

Boyer, R., (1986), La théorie de la régulation : une analyse critique, La Découverte.

Boyer, R., (2002-2003), « Variété du capitalisme et théorie de la régulation », L’Année de la régulation, $\mathrm{n}^{\circ} 6$, pp. 125-194.

Chesnais, F., (1997), La mondialisation du capital, Syros.

Cousy, J., (2003), « Les politiques publiques dans la mondialisation », L'économie politique, $\mathrm{n}^{\circ} 17$, janvier 2003, pp. 42-59.

Gorz, A., (1988), Métamorphoses du travail, Quête du sens, Galilée.

Héraud, J.-A. et Kahn, R., (2002), « L'action des collectivités entre développement endogène et exogène », Sciences de la société, $n^{\circ} 57$, octobre 2002.

Krugman, P., (1998), La mondialisation n'est pas coupable, La Découverte.

Laganier, R., Villalba, B. et Zuindeau, B., (2002), « Le développement durable face au territoire : éléments pour une recherche pluridisciplinaire », Développement Durable \& Territoires, $\mathrm{n}^{\circ} 1$ (www.revue-ddt.org).

Lipietz, A., (2001), Aménagement du territoire et développement endogène, Rapport au Conseil d'analyse économique, 18 janvier 2001.

Nieddu, M., (1999), « Secteurs et territoires, tour à tour problèmes et solutions dans les approches régulationnistes ", Séminaire Régulations Sectorielle et Territoriale, Paris, 22 mars 1999, www.upmf-grenoble.fr/irepd/regulations/Groupesderecherche/ RST/NIEDDU1.htm.

Michalet, C.-A., (1999), La séduction des nations, Economica.

Paquerot, S., (1996), L'État aux orties ?, Les éditions écosociété, Montréal.

Pecqueur, B., (2000), Le développement local, Syros.

Piore, M. et Sabel, C., (1984), The second industrial divide, Basic Book.

Porter, M., (1990), The competitive advantage of nations, The free press.

Stiglitz, J., (2002), La grande désillusion, Fayard.

Veltz, P., (2002), Des lieux et des liens, Éditions de l'Aube.

Veltz, P., (1996), Mondialisation villes et territoires. L'économie d'archipel, P.U.F.

Zuindeau, B., (Éd.), (2000), Développement durable et territoire, Presses Universitaires du Septentrion. 


\section{NOTES}

1. C'est la définition donnée par Martino Nieddu (1999, p. 4) pour caractériser le programme de recherche régulationniste concernant ce champ.

2. C'est la thèse de Krugman (1998).

3. Propos exprimés en agence régionale de développement.

4. Charzat M., Rapport au Premier ministre sur l'attractivité du territoire français, juillet 2001.

5. L'agence de promotion économique du Nord/Pas-de-Calais se nomme ainsi NFX (Nord France eXperts) et ses sites ne sont plus rédigés en français, tout comme l'adresse électronique est «locateinfrance.com ». De la même façon au niveau national, les IDE sont traités par Invest in France agency.

6. Dassault O., Rapport au Premier ministre sur l'attractivité du site France, janvier 2003.

7. Selon sa propre expression, La tribune, 31 janvier 2003.

8. Signe que cette localisation fiscale est entrée dans les pratiques, Sanofi-synthélabo lors de son assemblée générale 2003 se considère "plutôt exemplaire dans son impact français " pour n'avoir pas relocalisé son siège pour des motifs fiscaux. Cela s'insère de fait dans sa politique de Responsabilité Sociale d'Entreprise (cité dans Impact Entreprises, n³4, mai-juin 2003, p. 9).

9. C'est notamment la version du Conseil Régional Nord/Pas-de-Calais.

10. Une des traductions de cette logique de ressource est la spécialisation et s'oppose en fin de compte à tout ce qui découle d'une logique vivrière : le producteur local, étant soumis au prix du «marché mondial », ne lui survit pas. Une vision schématique darwinienne tend à s'imposer : seul le « plus efficace » peut survivre.

11. L'année record en matière de fusions-acquisitions est l'année 2000 (3540 milliards de dollars).

12. Définir la « nationalité » d'une firme est un exercice difficile mais exemplaire des différentes modalités de l'ancrage national. Néanmoins, il est plus facile pour une firme étrangère au tissu socio-politique de se désintéresser de l'effet collectif de son désengagement.

13. Cf. Alternatives économiques, février 2002 et février 2003.

14. La tribune, 31 janvier 2003.

15. Pollution, licenciement, déplacement rapide de capitaux spéculatifs... sont autant d'effets induits par des pratiques d'entreprises qu'elles ne prennent pas en charge et qui restent ainsi à gérer par la collectivité.

16. Une importante littérature est produite dès 1977, pour une synthèse, voir Benko et Lipietz, 1992.

17. Notion proposée en 1981 par Stöhr W. et Taylor D., Development above or below, Wiley.

18. Economiste, Alain Lipietz est porte-parole des Verts, un temps Conseiller Régional Ile-deFrance, puis actuellement député européen et conjointement membre du Conseil d'Analyse Économique mis en place par Lionel Jospin lorsqu'il était Premier ministre. La dimension critique et de projet de son rapport au CAE constitue un exemple des échanges entre terrain politique et terrain de recherche.

19. Bernard Pecqueur, enseignant chercheur à l'Université Grenoble II, publie régulièrement sur les thématiques du développement local, il est conseiller municipal de Grenoble et président de la commission « développement économique et emploi ».

20. Dans nombres de positions critiques, le responsable est ailleurs : FMI, Bruxelles, l'OMC ou les FMN, quand bien même des mandats publics régissent les décisions des hauts fonctionnaires dans les instances internationales. 


\section{RÉSUMÉS}

L'article montre comment la logique déréglementaire de mise en concurrence et de mise en management qui frappe les services publics frappe les territoires de façon similaire. L'action et les marges de manœuvre des pouvoirs publics sont remises en cause. La pensée du territoire en concurrence pousse à la constitution d'avantages concurrentiels faisant primer l'attraction des capitaux plutôt que le développement endogène. L'article distingue trois configurations. La valorisation quasi-publicitaire du territoire s'insère dans perspective de marketing territorial. Le dumping social met en avant le prix du territoire (niveau des salaires, flexibilité du travail, fiscalité). À ces modèles de rivalité s'oppose une politique de valorisation des compétences du territoire par le développement de biens publics (infrastructures en communication, formation, diffusion de technologie) qui deviennent la condition de l'attractivité du territoire.

This paper shows how the logic of deregulation, which creates competition, and the introduction of management methods in the public sector affect territories in the same way. Public policies are restricted by this change. Putting territories in competition with each other creates competitive advantages that attract capital rather than inciting endogenous development. The paper proposes three configurations. First, valorizing the territory through an advertising campaign is a form of territorial marketing. Second, social dumping brings to the fore the price of the territory (wage levels, flexibility, taxation). Third, and in opposition to these competitive models, we propose a policy that valorizes the competences of the territory by developing public goods (communication infrastructure, education, the spread of technology). These competences then become key to attractiveness.

\section{INDEX}

Mots-clés : développement territorial, mondialisation, politique, dumping social

Keywords : territorial development, globalization, politics, social dumping

\section{AUTEUR}

\section{THOMAS LAMARCHE}

Gerico - Université Charles-De-Gaulle - Lille 3

Thomas Lamarche est économiste à l'Université de Lille 3, ses recherches et sa conception des questions économiques le conduisent à privilégier une approche interdisciplinaire ; c'est ainsi qu'il est associé au laboratoire GERICO. Ses thèmes de recherche s'articulent autour des services publics et de l'action publique. Partant de travaux sur la déréglementation, il s'intéresse actuellement à deux nouvelles déréglementations : le territoire et l'éducation (sous l'angle de la mondialisation et du changement technologique). 